\title{
Management of Vatarakta with Mustadi Yapan Basti: A Case Study
}

\author{
Case Report
}

\section{Devyani Sanghani1, Sangeeta H Toshikhane²*, Lal Ravi Sahu³, Kritika Thakur ${ }^{4}$}

\author{
1. PG Scholar, 2. Professor and HOD, 3. Assistant Professor, 4. PG Scholar
}

Department of Panchakarma, Parul Institute of Ayurved and Research, Parul University, Vadodara, Gujarat.

\begin{abstract}
Vatarakta is a disease mentioned in Ayurveda involving Vata Dosha imbalance affecting Rakta Dhatu, where the Vayu is exacerbated by long distance rides on animals such as elephants, camels, horses, and Rakta or blood is vitiated by the ingestion of Lavana, Amla, Katu, Kshara, etc. The Vata, whose passages are covered by Rakta further undergoes vitiation and further contaminates the Rakta or blood. Vayu's vitiated blood later burns the entire blood in the body and gravitates towards the limbs later. This vitiated Vata and Rakta is called Vatarakta. On the basis of etiopathology, Vatarakta may also be linked to Gout. A 52 years old female patient approached the OPD with the chief complaints of: Acute pain in all major and minor joint and Deformity on upper limb fingers in the last one year. The patient was given completely Ayurvedic medicines and Panchakarma therapies for 10 days, and Only Ayurvedic medicines on discharge were later moved and the findings were noticeably seen. Hence this study was taken to prove that Ayurvedic management with Mustadi Yapana Basti has remarkable results on Vatarakta (Gout). Observations and outcomes were drawn on the basis of measurement criteria. During analysis, discussion was carried out on the basis of whole findings. On the basis of the outcome, conclusions were taken.
\end{abstract}

Key Words: Vatarakta, Gout, Vata dosha, Rakta dhatu, Mustadi Yapan Basti.

\section{Introduction}

Vatarakta is a painful condition. When aggravated Vata is obstructed by aggravated Rakta, this obstructed Vata in vitiates the Rakta. This pathological state is known as Vata Shonitam or Vatarakta . Vatarakta is described in details in Brihatrayis. In Sushruta Samhita, Vatarakta is described in Vata Vyadhi adhyaya. Here in chiefly small joints of feet and hands are affected. On the basis of etiology and symptomatology Gout is similar to that of Vatarakta. Gout is also called metabolic arthritis. Gout is an abnormality of Uric acid metabolism that results in hyperuricemia, deposition of monosodium urate crystal in joints, soft tissue and renal tubules. (1)

There are 2 types of Vata-Rakta ie, Utthana Vatarakta (superficial) and Gambhira Vatarakta (deeper tissue type). (2)

Table 1: Showing symptoms of Uthana and Gambhira Vatarakta (3)

\begin{tabular}{|c|c|c|c|}
\hline \multicolumn{2}{|c|}{ Uttan vatarakta } & \multicolumn{2}{|c|}{ Gambhir vatarakta } \\
\hline Kandu & Toda & Swayathu & Daha \\
\hline Daha & Spurana & Stambha & Toda \\
\hline Ruka & Kujana & Arathi & Spurana \\
\hline Ауата & Shyava rakta twak & Tamra varna & $\begin{array}{l}\text { Paka } \\
\text { Ruk }\end{array}$ \\
\hline
\end{tabular}

* Corresponding Author:

Sangeeta H Toshikhane

Professor and HOD, Department of Panchakarma,

Academic Coordinator, Parul Institute of Ayurved,

Parul University, Vadodara,

Gujarat. India.

Email Id: drsangeetaj@gmail.com

\section{Case Study}

A 52-year-old female patient registered by the O.P.D. and I.P.D. number $20011322 / 201245$ on the date of 11-07-2020 came to the O.P.D. no. 105 of Parul Ayurveda Hospital, Parul University and got admitted in Panchakarma IPD ward. She presented herself with the following complaints.

Main complaint with duration: Acute pain in all major and minor joint and deformity on upper limb fingers- in the last one year.

Other complaints: No any associated complain seen.

History of present illness:Patient was normal before 1 year and then gradually developed above symptoms occurs due to sedentary life style. So, for proper treatment she came to Parul Ayurveda Hospital, Parul University.

Family History: No any Family Illness of such History to any relatives.

Past History: No any past illness.

Table 2: Showing Personal history

\begin{tabular}{|l|l|}
\hline Appetite & normal \\
\hline Bowel & constipated / irregular (sometimes) \\
\hline Micturition & regular (4/2- Day/Night) \\
\hline Sleep & Disturbed \\
\hline Diet & Veg. \\
\hline Addiction & Tea- 2 times a day \\
\hline
\end{tabular}


Table 3: Showing General physical examination

\section{General condition} BP

PR

RR

Tongue

Temperature

Nourishment and Built

Lymph nodes

Pallor

Icterus

Cyanosis

Clubbing

Table 4: Showing Ashtavidh pariksha

\begin{tabular}{|l|l|}
\hline Nadi & Gati $-74 / \mathrm{min}$ \\
\hline Mutra & $\mathrm{D} / \mathrm{N}-4 / 2$ times \\
\hline Mala & Vibandhita Mala (sometimes) \\
\hline Jivha & Liptha \\
\hline Shabda & Prakrita \\
\hline Sparsha & Ushna Sparsa in affected Sandhis \\
\hline Druk & Prakrita \\
\hline Akriti & Madhyama \\
\hline
\end{tabular}

Table 5: Showing Dashvidh Pareeksha

\begin{tabular}{|l|l|}
\hline Prakriti & Vata Pitta \\
\hline Vikriti & $\begin{array}{l}\text { Vata- Shula, Pitta- Daha, Kapha- } \\
\text { Sthamba (fingers) }\end{array}$ \\
\hline Sara & Rasa, Rakta Sara \\
\hline Samhanana & Madhyama \\
\hline Pramana & Madhyama \\
\hline Satva & Madhyama \\
\hline Satmya & Shad Rasa Satmya \\
\hline Ahara Shakti & $\begin{array}{l}\text { Abhyavarana shakti: Madhyama } \\
\text { Jarana Shakti: Avar }\end{array}$ \\
\hline Vyayama Shakti & Madhyama \\
\hline Vayah & Yuva \\
\hline
\end{tabular}

Table 6: Showing Systemic Examination

Respiratory System Lung fields are clear, Air entry is good

Cardiovascular System S1S2 clear, No Murmurs

Gastrointestinal Tract P/A- Soft, Normal bowel sounds,

No Organomegaly.

Central Nervous Conscious and oriented,

System cooperative

Table 7: Showing Samprapti Ghataka

\begin{tabular}{|l|l|}
\hline Dosha & Vata, Pitta (Rakta) \\
\hline Dushya & Rakta, Twaka, Mamsa \\
\hline Adishtana & Rasavaha Srotas, Sandhi, Twaka, Mamsa \\
\hline Srotas & Raktavaha \\
\hline Agni & Mandagni \\
\hline Ama & Sama \\
\hline Srotodushti & Sanga Pashchat Vimarg Gamana \\
\hline Udbhawastana & Pakwashaya \\
\hline Vyakta Sthana & Sarva Sandhi \\
\hline Sadhya Asadhyata & Kruccha Sadhya \\
\hline Roga Marga & Madhyama (Twak, Mamsa, Asthi, Sandhi) \\
\hline
\end{tabular}

\section{Samprapti: (4)}

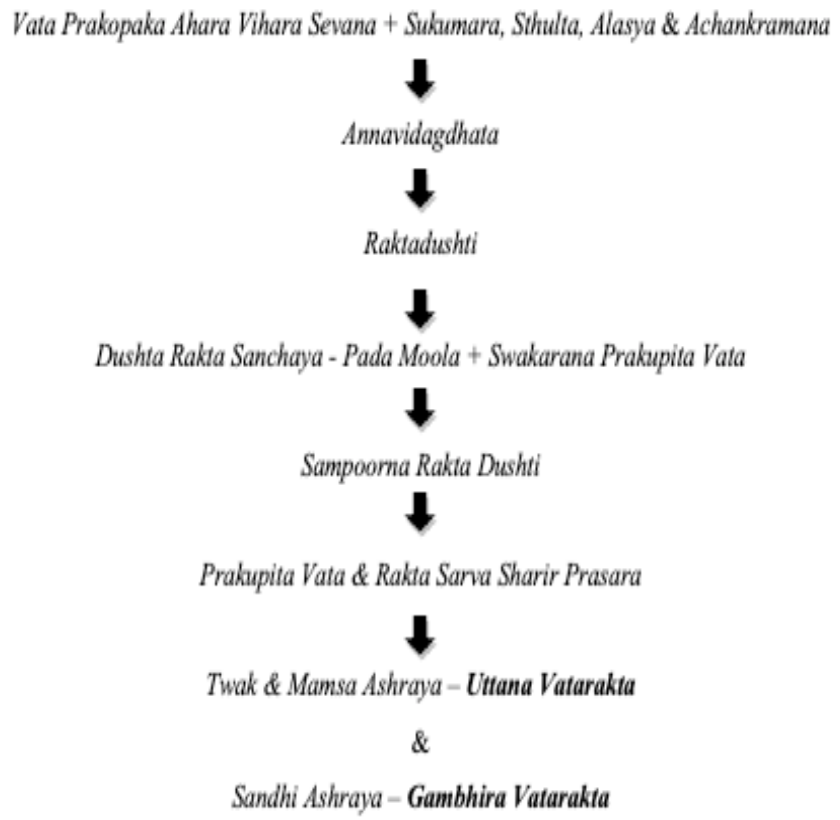

Table 8: Showing gradation of symptoms according to WHO scoring pattern (5)

\begin{tabular}{|l|l|l|l|l|}
\hline Symptom & Swelling & Discoloration & $\begin{array}{l}\text { Burning } \\
\text { sensation }\end{array}$ & \multicolumn{1}{|c|}{ Pain } \\
\hline Grade 0 & $\begin{array}{l}\text { No } \\
\text { swelling }\end{array}$ & $\begin{array}{l}\text { Normal } \\
\text { colouration }\end{array}$ & $\begin{array}{l}\text { No } \\
\text { burning }\end{array}$ & No pain \\
\hline Grade 1 & $\begin{array}{l}\text { Slight } \\
\text { swelling }\end{array}$ & $\begin{array}{l}\text { Near to } \\
\text { normal which } \\
\text { look like } \\
\text { normal from } \\
\text { distance }\end{array}$ & $\begin{array}{l}\text { Mild } \\
\text { burning }\end{array}$ & Mild pain \\
\hline Grade 2 & $\begin{array}{l}\text { Moderate } \\
\text { swelling }\end{array}$ & $\begin{array}{l}\text { Reddish } \\
\text { colouration }\end{array}$ & $\begin{array}{l}\text { Moderate } \\
\text { burning }\end{array}$ & $\begin{array}{l}\text { Moderate } \\
\text { pain but no } \\
\text { difficulty in } \\
\text { moving }\end{array}$ \\
\hline Grade 3 & $\begin{array}{l}\text { Severe } \\
\text { swelling }\end{array}$ & $\begin{array}{l}\text { Slight reddish } \\
\text { black } \\
\text { discolouration }\end{array}$ & $\begin{array}{l}\text { Severe } \\
\text { burning }\end{array}$ & $\begin{array}{l}\text { Slightly } \\
\text { difficulty in } \\
\text { moving due } \\
\text { to pain }\end{array}$ \\
\hline Grade 4 & - & $\begin{array}{l}\text { Blackish } \\
\text { discolouration }\end{array}$ & $\begin{array}{l}\text { Much } \\
\text { difficulty }\end{array}$ \\
\hline
\end{tabular}

Table 9: Showing Treatment Plan

\begin{tabular}{|c|c|c|c|}
\hline & Karma & Dravya & Days \& Action \\
\hline 1 & $\begin{array}{l}\text { Sarvanga } \\
\text { Parisheka }\end{array}$ & Dashmoola Kashaya & \multirow[t]{2}{*}{$\begin{array}{l}\text { For } 3 \text { days } \\
\text { (Shothaghna) }\end{array}$} \\
\hline 2 & Sthanik Lepa & Dashanga Choorna & \\
\hline 3 & Siravedha & & $\begin{array}{l}\text { On 3rd day } \\
\text { (Raktashodhana) } \\
\text { Kshipra Marma }\end{array}$ \\
\hline 4 & $\begin{array}{l}\text { Sthanika } \\
\text { Abhyanga }\end{array}$ & Murchita Tila Taila & \multirow[t]{3}{*}{ After $3^{\text {rd }}$ day } \\
\hline 5 & Swedana & Baspa Sweda & \\
\hline 6 & Basti & Mustadi Yapana Basti & \\
\hline
\end{tabular}

Basti plan:

1.Niruha Basti - Mustadi Yapana Basti

2.Anuvsana Basti-Murchita Tila Tila 
Table 10: Ingredients of Niruh Basti: (6)

1 Makshik $80 \mathrm{ml}$

2 Lavana $10 \mathrm{gm}$

3 Sneha Murchita Tila Taila $-40 \mathrm{ml}+$ Murchita GoGhrit $-40 \mathrm{ml}=80 \mathrm{ml}$

4 Kalka Putiyavanyadi Choorna

5 Kwatha Mustadi Ksheer Paka $=350 \mathrm{ml}$

Anuvasana Basti: Murchita Tila Taila: $80 \mathrm{ml}$

Table 11: Showing Basti schedule

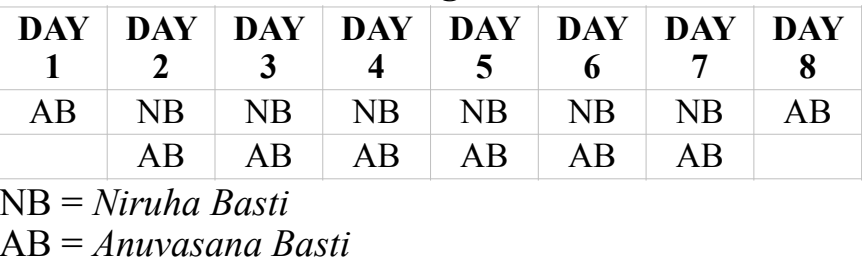

Table 12: Showing Shaman Aushadh: (After treatment below medicines are given)

\begin{tabular}{|c|c|c|c|c|c|}
\hline $\begin{array}{l}\text { Sr. } \\
\text { No }\end{array}$ & Medicine & Dose & Duration & Anupana & Action \\
\hline 1 & $\begin{array}{l}\text { Trikatu } \\
\text { Choorna }\end{array}$ & $3 \mathrm{gm}$ & $\begin{array}{l}3 \mathrm{gm} \\
-3 \mathrm{gm} \\
-3 \mathrm{gm}\end{array}$ & $\begin{array}{l}\text { Lukewarm } \\
\text { water }(\mathrm{B} / \mathrm{F})\end{array}$ & $\begin{array}{l}\text { Agnivardhaka, } \\
\text { (B.R. } \\
\text { Paribhasha } \\
\text { Prakarana) }\end{array}$ \\
\hline 2 & $\begin{array}{l}\text { Amapachana } \\
\text { Vati }\end{array}$ & $\begin{array}{l}250 \\
\text { gm }\end{array}$ & $2-2-2$ & $\begin{array}{l}\text { Lukewarm } \\
\text { water }(\mathrm{B} / \mathrm{F})\end{array}$ & Amapachana \\
\hline 3 & $\begin{array}{l}\text { Erand } \\
\text { Bhrushta } \\
\text { Haritaki }\end{array}$ & $\begin{array}{l}500 \\
\mathrm{mg}\end{array}$ & $0-0-3$ & $\begin{array}{l}\text { Lukewarm } \\
\text { water }(\mathrm{A} / \mathrm{F})\end{array}$ & $\begin{array}{l}\text { Vatanulomana } \\
\text { (Brihat } \\
\text { Nighantu } \\
\text { Ratnakara) \& } \\
\text { Bhavaprakasha }\end{array}$ \\
\hline 4 & $\begin{array}{l}\text { Kaishor } \\
\text { Guggulu }\end{array}$ & $\begin{array}{l}250 \\
\mathrm{mg}\end{array}$ & $2-2-2$ & $\begin{array}{l}\text { Lukewarm } \\
\text { water (A/F) }\end{array}$ & $\begin{array}{l}\text { Tridoshaghna, } \\
\text { Rasayana, } \\
\text { Vatarakta } \\
\text { Adhikar } \\
\text { (Sharangdhara } \\
\text { Samhita } \\
\text { Madhyama } \\
\text { Khanda } \\
7 / 70-81 \text { ) }\end{array}$ \\
\hline & $\begin{array}{l}\text { Amruttottra } \\
\text { Kashaya }\end{array}$ & $\begin{array}{l}15 \\
\mathrm{ml}\end{array}$ & $\begin{array}{l}15 \mathrm{ml}-0- \\
15 \mathrm{ml}\end{array}$ & $\begin{array}{l}\text { (B/F) with } \\
\text { equal } \\
\text { quantity of } \\
\text { water }\end{array}$ & $\begin{array}{l}\text { Shoolahara, } \\
\text { Jwarahara, } \\
\text { Shothahara } \\
\text { (SahasraYoga } \\
\text { Kashaya } \\
\text { Prakarana 30) }\end{array}$ \\
\hline
\end{tabular}

$(\mathrm{B} / \mathrm{F})=$ Before Food

$(\mathrm{A} / \mathrm{F})=$ After Food

\section{Observation}

The patient had started improving during hospital stay and at the end of the Basti Chikitsa, there was no any other complication seen. So, it needs time to cure but with Ayurvedic treatment the patient got symptomatic relief.
Table 13: Showing changes in symptoms before and after treatment

\section{Symptoms (U/L Before treatment After treatment fingers)}

\begin{tabular}{|l|l|l|}
\hline Swelling & 3 & 1 \\
\hline Discoloration & 2 & 1 \\
\hline Burning sensation & 3 & 1 \\
\hline Pain & 3 & 1 \\
\hline
\end{tabular}

Table 14: Showing changes in investigations

\begin{tabular}{|l|c|c|}
\hline INVESTIGATION & B.T. & A.T. \\
\hline 1.Hemoglobin & $12.8 \mathrm{gm} \%$ & $13 \mathrm{gm} \%$ \\
\hline 1.CBC & Normal & Normal \\
\hline 1.E.S.R. & $42 \mathrm{~mm} / \mathrm{hr}$. & $32 \mathrm{~mm} / \mathrm{hr}$. \\
\hline 1.Urine (Routine/Micro) & Normal & Normal \\
\hline 1.RBS & $100 \mathrm{mg} / \mathrm{dl}$ & $98 \mathrm{mg} / \mathrm{dl}$ \\
\hline 1.S- Uric acid & $5.4 \mathrm{mg} / \mathrm{dl}$ & $5.0 \mathrm{mg} / \mathrm{dl}$ \\
\hline
\end{tabular}

\section{Discussion}

Mode of Action of Snehana (7)

It helps for the Gati of Vata, Gaatra Mardavata and remove Srothorodha.

\section{Mode of Action of Swedana (8)}

It helps for Srothoshuddhi and Ama Pachana, that relieves stiffness, eliminates Kleda, and achieves lightness.

\section{Mode of Action of Basti}

Basti is that the best therapy to regulate the Vata and thus it controls the Pitta and Kapha also. The Tikshna Guna of Basti helps in overcoming the Sroto Dushti resulting because of Sanga. Thus, Basti Dravya after about to large and little intestine get absorbed, thanks to Laghu, Ushna, Tikshna, and Ruksha Guna of Dravyas, it breaks the obstruction and expel out the morbid material from everywhere the body. Vatarakta located in Paani (hands) is caused by upward Dosha and in Paada (feet) is because of downward Dosha. (9)

\section{Mode of Action of Ksheera Basti}

Milk is emulsion or colloid of butterfat globules within a water-based fluid that contains dissolved carbohydrates and protein aggregates with minerals. one in every of the important factors about the milk is that it contains calcium; it is absorbed without the assistance vit- D, Under the influence of lactose within the distal intestine via the paracellular route. This promotes the absorption of calcium and provides simultaneous intake of phosphorus that it essential for bone deposition. Hence Ksheer Basti have a large and effective applicability in Asthivaha Sroto Vikara.

\section{Conclusion}

Vatarakta has become a typical disease. If we pass, it is often derived that sedentary day today affaires together with unhealthy eating habits are the foundation cause behind this. The treatment and medicine assure permanent cure together 
with maintaining physical and mental state of the patient.

On understanding proper Nidana, Lakshana and Samprapti of Vatarakta one can fine keep it under the heading of Vata Vyadhi and treat it successfully with Panchakarma treatment. With proper understanding of Dosha, Dushya and Vyadhi Awastha we are able to manage Vatarakta. by following advanced Panchakarma treatment, here patient got relief from symptoms within 10 days. The treatment given to the patient mainly Basti is Kaphavatahara or Pittavatahara. mainly used for Deepana Paachana and Rakta Prasadana with Shoolahara and Shothhara Karma. The patient had symptomatic relief and therefore the laboratory findings of Serum acid had lowered significantly. Therefore, the given Ayurvedic treatment was successfully in curing the disease without landing into further complications.

\section{References}

1. 1. T Mahesh Babu, A. Vijayalakshmi, V Narasimha, Ayurvedic Management of Vatarakta w.s.r to gout-A case study, International Ayurvedical Medical Journal, (April,2017) 5(4).

2. 2.Yadavaji Trikamaji, editor, commentary: Ayurveda Deepika of Chakrapani on Charaka Samhita of Charaka, Chikithsasthana, chapter 29,verse no. 19, Varanasi; Chowkhambha Krishnadas Academy;p.628.

3. Yadavaji Trikamaji, editor, commen-tary:Ayurveda Deepika of Chakrapani on CharakaSamhita of Charaka, Chikithsasthana, chapter 29,verse no. 19-21, Vara-nasi; Chowkhambha Krishnadas Academy;p.628.

4. Ajay Kumar Sharma, Kaya Chikitsa - Hindi, Dwitiya Bhaga, chapter 63, ED 2014, Pub Chowkhambha Orientalia, pg.912.

5. Developing guidelines for clinical research methodology in Ayurveda, Proff. M. S Baghel, Dr. Rajagopala S. -WHO / TRM guidelines, 2000 downloaded from www.sho.int/ entity/hiv/amds / WHOTCM2005.1 OMS.pdf on 24.06.2006, New Delhi.

6. Priya Varta Sharma, Charak Samhita, Vol. 1, Charak Samhita, Siddhi Sthana, Chapter 12/16-1, Chaukhamba Sankrit Pratishthana,pg. 181.

7. Kaviraj Ambika Dutta Shastri(editor). Sushruta Samhita, Vol..I Sushruta. Chikitsa Sthana,Chapter 31, pg. 335.

8. Bhavaprakasha of Bhava Mishra the vidyotini Hindi, Commentary Notes, Introduction, editor Shri Brahma Shankara Mishra, Chaukhambha Sankrit Sansthan Varanasi Chap.29/72,306.

9. Kaviraj Ambika dutta Shastri (editor). Sushruta Samhita,Vol.I. Sushruta. Chikitsa Sthana, Chapter 5 verse 7, pg. 210. 